\title{
Description of a new species and new faunistic records of the genus Anomala Samouelle (Coleoptera, Scarabaeidae, Rutelinae) from China and neighboring regions
}

\author{
With 51 figures
}

FA-LeI WANG ${ }^{1}$ and CARSTEN Zorn ${ }^{2,3}$

1 Engineering Research Center for Forest and Grassland Disaster Prevention and Reduction, Mianyang Normal University, 166 MianxingWest Road, Mianyang, Sichuan 621000, P. R. China. - 531609816@qq.com.

2 Rostocker Straße 1a, 17179 Gnoien, Germany. - czorn70@hotmail.com

3 Corresponding author

Published on 2021-06-30

DOI:10.21248/contrib.entomol.71.1.147-159

\section{Abstract}

Anomala xiongi WANG \& ZorN spec. nov. is described from Yunnan, China. This new species can be distinguished from all other known Anomala species by conspicuous long erect setae rising from the primary costae of elytra. Additionally, ten new distributional records of Anomala species from China and neighboring countries are provided.

\section{Key words}

Oriental region, Palaearctic region, Asia, beetles, Insecta, Coleoptera, taxonomy, new distributional records

\section{Zusammenfassung}

Anomala xiongi WANG \& ZoRn spec. nov. wird aus Yunnan, China, beschrieben. Diese neue Art unterscheidet sich von allen anderen bekannten Anomala-Arten durch lang abstehende Haare auf den primären Rippen der Elytren. Darüber hinaus werden neue Funde von zehn Anomala-Arten aus China und angrenzenden Ländern präsentiert.

\section{Nomenclatural act}

Anomala xiongi WANG \& ZoRn spec. nov. - urn:lsid:zoobank.org:act:F10AD66D-BEF8-41E2-B22D-42D0492D9E95

\section{Introduction}

Anomala Samouelle, 1819 is the most species-rich genus in subfamily Rutelinae, with approximately 1300 described species (KRAjČík 2012). So far, nearly 220 species have been recorded in China (KRAJčík 2012; ZoRN \& BEZDĚK 2016; ZoRN et al. 2017). During the past decade, eight Anomala species were described from
China (Zorn 2011; Prokofiev 2015; Huang \& Wang 2019; ZHAO 2019). Based on an examination of recently collected Anomala material from southern China and neighboring regions, we describe one new species from China and present new distributional records for ten known species. 


\section{Material and Methods}

Morphological terminology used in this study is based on Zorn (2007). The body length was measured from the apex of the clypeus to the apex of the pygidium. Type specimens of the new species described in this paper bear the following labels: 1) printed type designation label: "HOLOTYPE (or PARATYPE) / Anomala xiongi new species, det. Wang \& Zorn 2019"; 2) red "Holotype" or "Paratype" label; 3) collecting data labels. The collecting data of the type material are cited verbatim from the labels, individual labels are separated by a slash (/). All photographs were edited in Adobe Photoshop ${ }^{\circledR}$ CC 2018. We use the following abbreviations for collections:

AHPC Collection Andreas Hetzel, Münster, Germany CCPC Collection Chang-Chin Chen, Tianjin, China

CWNU China West Normal University, Nanchong, China

CZGG Collection Carsten Zorn, Gnoien, Germany

CZMZ Collection Ming-Zhi Zhao, Guangzhou, China

FLWC Collection Fa-Lei Wang, Chongqing, China

HNHM Hungarian Natural History Museum, Budapest, Hungary

MFNB Museum für Naturkunde Berlin, Leibniz-Institut für Evolutions- und Biodiversitätsforschung, Berlin, Germany

MSPC Collection Matthias Seidel, Hamburg, Germany

MNHN Museum national d'Histoire naturelle Paris, France

MYTC Mianyang Normal University, Sichuan, China

NMEG Naturkundemuseum Erfurt, Erfurt, Germany

NMPC National Museum (National History), Prague, Czech Republic

PLPC Collection Paul Lago, Oxford, Mississippi, USA

PPPC Collection Petr Pacholátko, Brno, Czech Republic

SNSD Senckenberg Naturhistorische Sammlungen Dresden, Dresden, Germany

SYSU The Museum of Biology, Sun Yat-sen University, Guangzhou, China

ZMH Zoologisches Museum Hamburg, Centrum für Naturkunde, Germany

\section{Description of the new species}

Anomala xiongi WANg \& ZoRN, spec. nov. urn:lsid:zoobank.org:act:F10AD66D-BEF8-41E2-B22D-42D0492D9E95 (Figs 1-12)

Type locality: China, Yunnan, Lincang, Yunxian County.

Type material: Holotype: ơ (MYTC), China, Yunnan, Lincang, Yunxian County, Manwan, Shuibatou / $24^{\circ} 70^{\prime} 17^{\prime \prime N}, 100^{\circ} 36^{\prime} 31^{\prime \prime E}$, alt. $1791 \mathrm{~m}$, VII-VIII.2018, Zi-Chun Xiong leg. Paratypes: $80^{\top} 0^{\top}, 8$ 우 우 (MYTC), same data as the holotype; $3 o^{\top} o^{\pi}$ (MYTC), China,
Yunnan, Lincang, Yunxian County, Manwan, Shuibatou / 24⒎'17"N, 100³6'31"E, alt. $1791 \mathrm{~m}$, VII-VIII.2017, Zi-Chun Xiong leg.; $3 \sigma^{\star} \sigma^{*}, 3$ 우 (FLWC), China, Yunnan, Lincang, Yunxian County, Manwan, Shuibatou

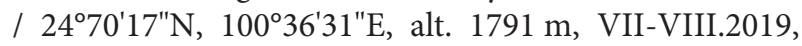
Zi-Chun Xiong leg.; 4 우 우 (MYTC), China, Yunnan, Lincang, Yunxian County, Manwan, Shuibatou / $24^{\circ} 70^{\prime} 17^{\prime \prime N}, 100^{\circ} 36^{\prime} 31^{\prime} \mathrm{E}$, alt. $1791 \mathrm{~m}$, VII-VIII.2019, Zi-Chun Xiong leg.; $10^{\star}, 1$ (MYTC), China, Yunnan, Dehong, Yingjiang County, Mangyun / 24 $45^{\prime} 55^{\prime \prime} \mathrm{N}$, 98 51'57"E, alt. $943 \mathrm{~m}$, VII-VIII.2019, Shao-Fu Chen leg.; $3 \sigma^{\star} o^{\star}, 2$ 우 우 (CZMZ), China: Yunnan, Lincang, Cangyuan County, Nangunhe River, Wengding Preserve Station, 1800 m, 2019.V.1-6, Yu-Chen Zheng leg.; $10^{*}$, 2 우 우 (CZMZ), China: Yunnan, Lincang, Yunxian County, Manwan, Shuibatou Village, 2014.VII-VII, light trap, Zi-Chun Xiong leg.; 1 ㅇ (CCPC), China, Yunnan, Lincang, Yongde, Yalian, Damaidi Village, 2012.V.8, X. D. Yang leg.

Description (male, holotype): Length: $13.4 \mathrm{~mm}$; width: $6.5 \mathrm{~mm}$; body elongate ovoid, moderately convex.

Coloration (Fig. 1): Body light brown except for head and legs; head medium brown; anterior margin of clypeus, frontoclypeal suture, ocular canthus and posterolateral portion of vertex blackened; antenna light brown; pronotum with small, vague brown spots near middle of each side; anterior and posterior margin blackened; scutellum with blackened margin; elytra with all margins narrowly blackened except for the suture with a broader blackened area reaching the subsutural interstice; pygidium with a vague, blurry blackish patch near middle of anterior margin; membrane of posterior margin of each abdominal ventrite dark brown; legs predominantly red-brown, with the distal portion of tarsomeres and tibiae blackened, major part of femurs light brown. Head. Clypeus subtrapezoidal, disc transversely punctate, anterior margin rounded, weakly reflexed; frons with deep punctures, which are rugose anteriorly and separated posteriorly. Ratio of interocular width/head width approximately 0.55 ; antenna long, with pale hairs, antennal club length equals combined length of antennomeres 2-6.

Thorax: Pronotum approximately 1.8 times as wide as long; disc with vague and discontinuous impressed midline; with moderately dense, moderately fine, deep, irregular punctation, somewhat denser and smaller laterally; anterior angles acute and protruding, posterior angles subrectangular, rounded off; sides of pronotum weakly converging anteriad in middle, slightly sinuate in anterior half, subparallel in posterior half; lateral margins with several long setae; anterior marginal line complete, with several short setae near anterior angles which gradually become more distant toward middle; basal marginal line complete and distinct, ending near posterior angles.

Scutellum triangular, with the sides slightly curved; punctures denser and smaller laterally than those at disc; lateral margins smooth; posterior corner sharp. 
Elytra approximately 1.5 times longer than wide; elytral surface smooth and shining, striate, strial punctures large and deep; primary striae regular, sulcate, punctures sometimes adjacent to each other; subsutural interstice with irregular punctation anteriorly and a regular secondary stria in the posterior $2 / 3$; second interstice with single secondary stria anteriorly which is irregularly doubled in the posterior $3 / 4$; third interstice with somewhat irregular secondary stria; primary costae and lateral margin with long erect setae, setae of lateral margin distinctly more numerous than those of primary costae; umbones prominent, apico-sutural angle subrectangular, slightly rounded; epipleuron broad near humerus, narrow, ending in posterior third, flat; lateral margins with numerous short yellow setae, sparse in anterior and posterior fourth, dense in elytral mid length.

Ventral thoracic surface with dense, round, large punctures, and dense, moderately long semierect setosity; disc with very fine, sparse punctures, almost glabrous. Pygidium (Fig. 9) triangular, convex; disc with moderately coarse, somewhat transverse punctures, indistinct along anterior margin, partly confluent laterally; posterior marginal line complete; with very sparse long, erect setae.

Legs: Protibia tridentate, apical and middle tooth rather long, basal tooth short, obtuse; with a rather long inner spur; mesotibia weakly fusiform; metatibia very fusiform; outer surface smooth with sparse fine punctures with a single oblique row of rigid spines; with two strong terminal spurs, the upper one 2 times longer than the lower; all tarsi rather long and slender, inner protarsal claw not clefted; metatarsus much longer than metatibia; outer mesotarsal claw clefted, inner mesotarsal claw and metatarsal claws long and simple.

Abdominal ventrites (Fig. 10) with rather sparse, transverse fine punctures in the middle, punctures become gradually denser and larger laterad; with a transverse row of moderately long, yellow setae, interrupted in the middle; with several additional setae laterally; sides carinate.

Aedeagus: parameres simple, short; ventral plate short, hook-shaped in lateral view. As in Figs 3-6.

Female (Fig. 2): Body slightly stouter; sides of pronotum more strongly curved, subparallel in posterior half; blackish markings much more extended compared to males: pronotum with small black spot near middle of each lateral margin and with two large trapezoidal spots covering the middle of anterior disc, encompassing also the black anterior margin; basal margin of pronotum blackish; major part of central elytral disc and humeral umbones black; protarsus slightly shorter than in male; inner protarsal claw and outer mesotarsal claw apically clefted; erect setae of elytra somewhat longer than in male.
Variability of body size: Male body length: 12.5$14.3 \mathrm{~mm}$, body width: $5.6-6.7 \mathrm{~mm}$; female body length: 13.4-14.9 mm, body width: $6.1-7.2 \mathrm{~mm}$.

Diagnosis: Anomala xiongi WANG \& ZoRN, new species can be distinguished from all other Anomala species by conspicuous long erect setae rising from the primary costae of elytra (Figs 1,2). This new species is most similar to A. fusitibia Lin, 1992, described from Yunnan and found in Laos and Thailand (see below). But, in addition to the aforementioned setae, A. xiongi also differs from A. fusitibia in the following characters: protibia tridentate, inner protarsal claw not cleft in male (Fig. 7).

Etymology: The new species is named after $\mathrm{Zi}$-Chun Xiong (Yunxian County, Yunnan), who collected the majority of the type material, including the holotype.

\section{New distributional records}

\section{Anomala ahrensi ZoRN, 2011}

Distribution: Vietnam, China (new country record): Yunnan.

Specimens examined: CHINA: Yunnan: $10^{\top}, 1$ 우 (FLWC), Jinping County, Honghe Pref., V-VI.2018, Yun-Chuan Xu leg.; 1 + (CCPC), Biaoshuiyan, Ma’andi, Jinping County, Honghe Pref., 2010.V.13 X. D. Yang leg.; 1 ơ (CCPC), Luobodi Village, Ma'andi, Jinping County, Honghe Pref., 2010.V.17, X. D. Yang leg.

Remarks: Anomala ahrensi was previously known only from northern Vietnam (ZoRN 2011, ZorN et al. 2017). Herein, we newly report this species from Yunnan, China.

\section{Anomala anguliceps ArRow, 1917 (Figs 13, 22-24)}

Distribution: Cambodia; China: Fujian, Guangdong, Guangxi, Sichuan, Yunnan, Zhejiang, Hunan (new Chinese provincial record); Laos; Myanmar; Thailand; Vietnam.

Specimens examined: CHINA: Guangdong: $4 \sigma^{\text {ते }}$, 5 우 우 (FLWC), Fenghuang, Fuyong, Baoan, Shenzhen, 18.V.2019, Ming-Yu Zhou leg. Hunan. 1 ㅇ (FLWC), Zhongzhou, Changsha, 09.VI.2011, Hao Xu leg. Sichuan: 1 ㅇ (FLWC), Luozong Mount, Puge County, VI.2016, Chao Zhou leg.; $10^{x}, 2$ ㅇ f (FLWC), Mujiashanzhai, Emei Mount, Leshan, 20-21.VII.2018, Lu Qiu leg.; $10^{\star}, 4$ 우 (CWNU), Hekou, Jiangyou, Sichuan, 16-17.VII.2009, Juan Li \& Xia Yan leg.; $20^{\star} \sigma^{*}$, 4 우 우 (CWNU), Wudu, Jiangyou, Sichuan, alt. $436 \mathrm{~m}$, 9.VII.2009, Ai-Ming Shi leg.; 1 o $^{\top}$ (CWNU), Meiguiyuan, Jiange, Sichuan, alt. 542 m, 7.VII.2009, Yong-Fu Wang \& 
Ru-Yi Li leg.; 1 ơ (CWNU), Shiqiao, Jianyang, Sichuan, 8.VII.2009, Ai-Ming Shi leg.; $10^{*}$ (CWNU), Chenghua District, Chengdu, Sichuan, 12.VIII.2017, Si-Yu Chen leg.; $10^{*}$ (CWNU), Mianyang, Sichuan, 7.VII.2017, Yu-Shuang Zhao leg.; $20^{\star} o^{\star}, 3$ 우 우 (CWNU), Qianfeng, Sichuan, 26.VI.2016, Xiang He leg. Yunnan: $20^{\star} \sigma^{*}$ (FLWC), Manjiu, Manwan, Yunxian County, Lincang, alt. 2000 m, 20.V.2015, Fa-Lei Wang leg.; $12 \sigma^{\top} \sigma^{\star}, 12$ 우 우 (FLWC), Manjiu, Manwan, Yunxian County, Lincang, alt. 2,000 m, VII.2017, Zi-Chun Xiong leg.; 11 o $^{\star}{ }^{*}$, 12 ㅇ ㅇ (FLWC), Manjiu, Manwan, Yunxian County, Lincang, alt. 2000, VII-VIII.2018, Zi-Chun Xiong leg.; $1 \sigma^{*}$ (FLWC), Baihualing, Longyang, Baoshan, VII.2014, Huang-Shun Xi leg.; 1 ㅇ (FLWC), Ailao Mount, Xinping County, Yuxi, VI.2014, Huang-Shun Xi leg.; 2 우 우 (FLWC), Baihualing, Longyang, Baoshan, V.2016, ZhiWei Dong leg.; $10^{\star}$ (FLWC), Tongbiguan, Yingjiang County, Dehong, 2.VI.2018, alt. 1345 m, Lu Qiu leg.; $3 \sigma^{\star} o^{\star}, 5$ ㅇ 우 (FLWC), Hulukou, Xima, Yingjiang County, Dehong, VI-VII.2018, Wei-Zong Yang leg.; 1 ㅇ (FLWC), Mangyun, Pingyuan, Yingjiang County, Dehong, 15-30. VI.2019, Gui-Chang Liu leg.; $1 \sigma^{\star}$ (FLWC), Tongbiguan, Yingjiang County, Dehong, 2.VI.2018, alt. $1345 \mathrm{~m}$, Lu Qiu leg.; 2 ㅇ ㅇ (FLWC), Nabang, Yingjiang County, Dehong, VIII.2018, Lu Qiu leg.; 1 † (FLWC), Meizihu, Simao, Puer, 9-11.V.2018, Lu Qiu leg.; $10^{\star}$ (FLWC), Yingjiang County, Dehong, 15-30.VI.2018, Peng Wang leg.; $10^{*}$ (FLWC), Zhenyuan County, Puer, VII.2018, Lu Qiu leg.

Remarks: This is a widely distributed species in South East Asia, which occurs in China, Cambodia, Laos, Myanmar, Vietnam and Thailand (Arrow 1917; Prokofiev 2014; Zorn \& BeZděK 2016; Zorn et al. 2017). ZORN \& BEZDĚK (2016) reported this species from the Chinese provinces of Fujian, Guangdong, Guangxi, Sichuan, Yunnan and Zhejiang. Herein, Hunan is a new provincial record for this species in China.

\section{Anomala anthracina ARRow, 1912} (Figs 14, 25-28)

Distribution: Bhutan; India: Sikkim; China (new country record): Xizang, Yunnan; Nepal (new country record).

Type material: Syntypes. $1 \sigma^{\star}(\mathrm{BMNH})$, Type / $\sigma^{\star} /$ Kurseong, Sikkim. R. P. Verschraeghen. 1911-218. / Kurseong Inde Verschraeghen 1904. 1908 / Anomala anthracina, Arrow Type [Arrow's handwriting]; 2 우 우 (BMNH), Kurseong, Sikkim. R. P. Verschraeghen. 1911-218. / Kurseong Inde Verschraeghen 1904. 1908; $10^{*}$ (BMNH), Co-type / MUSEUM PARIS SIKKIM HARMAND 1886 / Anomala anthracina Arr. [Arrow's handwriting]; 1 i (BMNH), Cotype / MUSEUM PARIS SIKKIM DARJEELING HARMAND 1890; 1 우 (BMNH), Mungphu / Atkinson Coll. 92-3 / Anomala anthracina, Arrow [Arrow's handwriting].
Other specimens examined: CHINA: Xizang: $10^{\star}$ (SYSU), Tibet, Bomi County, nr. 318 national road, 3003'54"N 95²11'23"E, 2300 m, 2018.VI.19, S. S. Wang $\&$ Z. L. Liang leg. Yunnan: $60^{\star} o^{\star}, 13$ 우 (FLWC), Gongshan County, 30.V.2018, Zheng Zhou leg.; 1 क (FLWC), Hulukou, Xima, Yingjiang County, VI-VII.2018, WeiZong Yang leg.; 1 ㅇ (FLWC), Mangyun, Pingyuan, Yingjiang County, VIII.2019, Gui-Chang Liu leg. INDIA: $10^{*}, 1$ ㅇ (CZGG), W Sikkim Pemayangste env., 19002080 m, 15-17.V.1998 leg. Fabrici \& Ahrens; 1 o (CZGG), India (Arunachal Pradesh), Tawang Ugelling, 19.VI.2014, K. Krause lgt.; 1 ㅇ (CZGG), India W. Bengal, Darjeeling env. 1800-2500 m, 8-10.V.1991, St. Jakl lgt. NEPAL: $1 \sigma^{*}$, 1 i (CZGG), Nepal east, Taplejung, 23-24.V.2013, lgt. E. Kučera.

Remarks: Anomala anthracina was formerly known only from Bhutan and India (Sikkim) (ARrow 1912a; Arrow 1917; Frey 1975; Sabatinelli 1997; Zorn \& BeZdĚK 2016). Here, it is reported from Xizang and Yunnan, China, and Nepal for the first time.

\section{Anomala bilobata ARrow, 1912 \\ (Figs 15, 29-32)}

Distribution: India; Myanmar; Nepal; China (new country record): Yunnan.

Specimens examined: CHINA: Yunnan: $7 \sigma^{\star} o^{\star}, 11$ ㅇ 우 (FLWC), Hulukou, Xima, Yingjiang County, Dehong, VI-VII.2018, Wei-Zong Yang leg.; $1 \sigma^{\star}$ (FLWC), Yingjiang township, Yingjiang County, Dehong, VI.2018, Yang Yu leg. NEPAL: 1 क (NMEG), Nepal, Prov: Narayani vic. Chandranigahaui $27^{\circ} 07^{\prime} 25^{\prime \prime} \mathrm{N}, 85^{\circ} 21^{\prime} 11^{\prime \prime E}, 130 \mathrm{~m}$, 02.V.2003, leg. A. Weigel HF/LF; $10^{\star}$ (NMEG), Nepal, Mechi/Taplejung, $9 \mathrm{~km}$ S Taplejung, Angbung Kabeli Khola (bridge), $450 \mathrm{~m}, 27^{\circ} 17^{\prime} 11^{\prime \prime N}, 8^{\circ} 43^{\prime} 21^{\prime \prime E}, 03 . V .2003$, leg. A. Weigel.

Remarks: Anomala bilobata was originally described from Bengal, India, and several localities in Myanmar (ARrow 1912b) and subsequently reported from Nepal (ZORN \& BEZDĚK 2016). It is here reported from Yunnan for the first time, representing a new country record for China.

\section{Anomala callifera OHAUs, 1937}

(Figs 16, 33-35)

Distribution: Myanmar; China (new country record): Yunnan.

Specimens examined: CHINA: Yunnan: 1 ㅇ (CZGG), China, W Yunnan prov., mts. $60 \mathrm{~km}$ E Tengchong, $2200 \mathrm{~m}$, 19-22.V.2006, S. Murzin \& I. Shokhin leg.; 1 ㅇ (CZGG), China, Yunnan prov., Gaoligongshan mts. $90 \mathrm{~km} \mathrm{~W}$ of 
Baoshan, 26-28.V.1995, lgt. S. Bečvář; 19 o o 28 ㅇ (FLWC), Dulongjiang, Gongshan County, 30.V.2018, Zheng Zhou leg.; $10^{\star}$ (FLWC), Tongbiguan, Yingjiang County, Dehong, 2.VI.2018, alt. 1345, Lu Qiu leg.; 2 우 우 (FLWC), Hulukou, Xima, Yingjiang County, Dehong, V-VII.2018, Wei-Zong Yang leg.; $1{ }^{\star}$ (FLWC), Mangyun, Pingyuan, Yingjiang County, Dehong, V-VII.2018, BaoYing Jin leg.

Remarks: Anomala callifera was previously known only from Myanmar (OHAus 1914a, 1937). In this article, we for the first time provide records from Yunnan, China.

\section{Anomala cantorioides PAULIAN, 1959}

(Figs 17, 36-39)

Distribution: Vietnam; China (new country record): Yunnan; Laos (new country record); Myanmar (new country record); Thailand (new country record).

Type material: Holotype. $\sigma^{\top}(\mathrm{MNHN})$, YEN-BAI Tonkin / Guy Babault / MUSẼUM PARIS 19 GUY BABAULT / Ohaus determ. Anomala cantori Hope [Ohaus's handwriting] / Anomala cantorioides n. sp. Type. [Paulian's handwriting] R. Paulian det. / HOLOTYPE / MNHN EC 1704.

Other specimens examined: China: Yunnan: $2 \sigma^{\star} o^{*}$ (CZGG), China, Yunnan, Xishuangbanna, Mengla, 1000 m, VI-VIII.2014, local collector lgt.; 1 o (CZMZ), Yunnan, South of Mengla, 2015.IX.10, leg. Huang; 1 ㅇ (CCPC), China, Yunnan, Mengla, Mohan $1100 \mathrm{~m}$, 2018.V.13-18, leg. C. C. Chen; 1 우 (NMEG), China, S-Yunnan, Xishuangbanna, $23 \mathrm{~km}$ NW Jinghong, vic. Na Ban, $21^{\circ} 09,49 \mathrm{E} 100^{\circ} 39,92 \mathrm{~N}, 730 \mathrm{~m}, 20 . \mathrm{V} .2008$, leg. A. Weigel. Laos: 2 ㅇ ㅇ (CZGG), Laos central, $70 \mathrm{~km}$ NE Vientiane, Ban Phabat env., N18 ${ }^{\circ} 16.1$ E103ำ10.9, 150 m, 27.IV.-1.V.1997, M. Štrba \& R. Hergovits leg.; 2 o $^{\top}$ (CZGG), Laos Luangphabang province, Mt. Phou Phakhao, Namtap vill., June 7-11.2008, local collector leg.; $10^{*}$ (PPPC), Laos, 4.V.1997. Louangnamtha pr.,

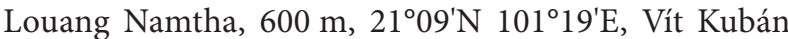
leg. Myanmar: $1 \sigma^{\top}(\mathrm{BMNH})$ Upper Burma Mt. Popa 600-1000 m, X.-.XI.37, leg. G. Heinrich. Thailand: $10^{\star}$ (PLPC), Thailand: Nan Province, Amphur Chiang Klang, $8 \mathrm{~km} \mathrm{~N}$ of Chiang Klang on Hwy 1080, $19^{\circ} 21^{\prime} \mathrm{N} 100^{\circ} 51^{\prime} \mathrm{E} ; 277 \mathrm{~m}$, lights; 20 April 2003, AV-5, Vitheepradit, Prommi, Setaphan; $10^{\top}$ (CZGG), Thailand, Chiang Dao, 600 m, 19² $4^{\prime} \mathrm{N} 98^{\circ} 55^{\prime} \mathrm{E}, 10-16$. V.1991, Vít Kubáň leg.; 1 † (CZGG), North Thailand, Chiang Dao, 10-20.VII.2004, R. Kocina lgt.; $1 \sigma^{\star}$ (CZGG), Thailand N., Mae Hong Son env., Thai/Burma border, 1300 m, 17-21.VI.1993, J. Schneider lgt. Vietnam: $10^{\star}$ (CZGG), NW Vietnam, Ninh Binh Prov., Dong Tam vill., Cuc Phuong N. P., $20^{\circ} 15^{\prime} \mathrm{N} 105^{\circ} 43^{\prime} \mathrm{E}, 03$. V.2019, $\mathrm{h}=90 \mathrm{~m}, \mathrm{D}$. Spiridonov leg.
Remarks: This species was previously known only from the type locality "Yen Bai" in northern Vietnam (Paulian 1959). Here we report new records for China, Laos, Myanmar, and Thailand.

\section{Anomala fusitibia LIN, 1992}

(Figs 18, 40-42)

Distribution: China; Laos (new country record); Thailand (new country record).

Specimens examined: CHINA: Yunnan: $1 \sigma^{\star}$ (FLWC), Manwan, Yunxian County, Lincang, VII.2017, Zi-Chun Xiong leg.; 1 o $^{*}, 1$ \& (FLWC), Manwan, Yunxian County, Lincang, VI-VII.2018, Zi-Chun Xiong leg.; 1 ๙ $^{\star}$ (FLWC), Xingguo, Pingyuan, Yingjiang County, Dehong,

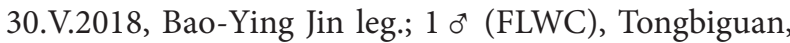

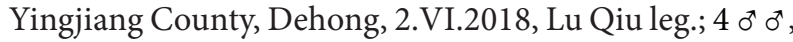
5 우 우 (FLWC), Xima, Yingjiang County, Dehong, 15-30. VI.2018, Peng Wang leg.; 1 ㅇ (FLWC), Zhenyuan County, Puer, VII.2018, Lu Qiu leg.; $2 \sigma^{\star} \sigma^{\star}, 4$ ㅇ (FLWC), Hulukou, Xima, Yingjiang County, Dehong, VI-VII.2018, Wei-Zong Yang leg.; 2 ๙ o $^{\star}, 3$ 우 우 (FLWC), Xima, Yingjiang County, Dehong, 15-30.VI.2018, Wei-Zong Yang leg.; $20^{\star} \sigma^{\star}, 1$ ㅇ (FLWC), Menglun Reservoir, Mengla County, Xishuangbanna, alt. 620 m, 15-17.V.2018, JianYue Qiu \& Hao Xu leg.; 1 o (FLWC), Nabang, Yingjiang County, Dehong, VIII.2018, Lu Qiu leg.; 1 ㅇ (FLWC), Mount Jinuo, Jinghong, 13-15.V.2018, Lu Qiu leg.; 2 ○ ठ (CZGG, NMPC), China: Yunnan prov., Gaolingong Mts. NNR, Baihualin vill., $1535 \mathrm{~m}, 25^{\circ} 17.7^{\prime} \mathrm{N} 98^{\circ} 48.1^{\prime} \mathrm{E}$, at light in village, J. Hájek \& J. Růžička leg. LAOS: $60^{\star} 0^{\star}$ (CZGG), Laos-N, Xieng Khuang prov., Nong Haet, $19^{\circ} 30^{\prime} \mathrm{N} 104^{\circ} 03^{\prime} \mathrm{E}, 29-31 . V .2001$, D. Hauck leg.; $10^{\star \top}$ (NMPC), Laos, Phongsaly prov. $21^{\circ} 41^{\prime} \mathrm{N}, 102^{\circ} 06^{\prime} \mathrm{E}$, Phongsaly env., 6-17.V.2004, 1500 m, Vít Kubáň leg.; 1 ㅇ (CZGG), Laos-NE, Hua Phan prov., $25 \mathrm{~km}$ SE Vieng Xai (by road), Ban Kangpabong env., $20^{\circ} 19^{\prime} \mathrm{N} 104^{\circ} 25^{\prime} \mathrm{E}$,

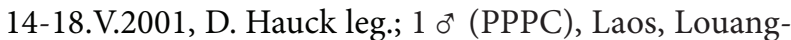
namtha prov., Namtha-MuangSing, $1200 \mathrm{~m}, 21^{\circ} 09^{\prime} \mathrm{N}$ 101¹9'E, 5-31.V.1997, Vít Kubáň leg. THAILAND: 1 o (CZGG), Thailand bor., Chiang Dao env., 17-19. IV.1995, leg. M. Snižek; 1 o*, 1 ㅇ (CZGG), Thailand bor., Chiang Dao env., 21.V.-4.VI.1995, leg. M. Snižek; $1 \sigma^{*}$ (PLPC), Thailand: Chiang Mai Prov., Doi Inthanon N. P., Nam Tok, Sob Ab, 18³1.775'N 98³6.488'E, 543 m, UV pan trap, 17 May 2004, Vitheepradit \& Prommi leg.; 1 ㅇ (PLPC), Thailand: Chiang Mai Prov., Doi Inthanon N. P., Nam Tok, Sob Ab, $18^{\circ} 31^{\prime} \mathrm{N} 98^{\circ} 36^{\prime} \mathrm{E}, 543 \mathrm{~m}$, 17. May 2004, Vitheepradit \& Prommi leg.; $10^{\top}$ (CZGG), Thai-N, Chiang Mai prov., Ban Sanpakia, $1400 \mathrm{~m}$, 1-19.V.1998, Ivo Martinů leg.; 1 o , 3 ㅇ ㅇ (CZGG), Thailand, Mae Hong Son prov., Soppong, $1500 \mathrm{~m}, 19^{\circ} 27^{\prime} \mathrm{N}$, 98²0'E, 7-12.V.1996, lgt. S. Bečvář; 2 o $^{\star} 0^{\star}$ (CZGG), Thai-N, Nan prov., Doi Pukha N.P. Headq., $1500 \mathrm{~m}$, $19^{\circ} 13^{\prime} \mathrm{N} 101^{\circ} 07^{\prime} \mathrm{E}, 22-26 . I V .1999$, D. Hauck leg.; 1 o $^{*}, 1$ ㅇ (CZGG), Thai-N, Mae Hong Son prov., SE Soppong, 
1500 m, 19²7'N 98²0'E, 23-27.V.1999, D. Hauck leg.; $4 \sigma^{\star} \sigma^{*}$ (CZGG, PLPC), Thailand, Phitsanulok prov., Phu Hin Rongkla N.P., UV pan trap at guest house, $1400 \mathrm{~m}, 17^{\circ} 00^{\prime} \mathrm{N} 100^{\circ} 59^{\prime} \mathrm{E}, 5$. May 2003, leg. Vitheepradit, Prommi, and Ferro; 1 o (CZGG), Thailand: Chiang Mai prov., Doi Inthanon N.P., Nam Tok Sob Ab, 543 m, $18^{\circ} 31^{\prime} \mathrm{N} 98^{\circ} 36^{\prime} \mathrm{E}, 17$. May 2004, leg. Vitheepradit \& Prommi; 4 우 우 (PLPC), Thailand, Loei Province, Na Haew Nat. Park, 978 m, light at guest house, 24.IV.2003, leg. Vitheepradit, Prommi, Setaphan.

Remarks: This species was described by LiN (1992) from Yunnan, China and subsequently also reported from the "Oriental Region" without further details in ZoRN \& BEZDĚK (2016). Herein, a considerable number of specimens were examined; those from Laos and Thailand represent new country records.

\section{Anomala kuatuna (MACHATSCHKE, 1955) (Figs 19, 43-45)}

Distribution: China: Fujian, Guangdong, Guangxi, Zhejiang, Guizhou (new Chinese provincial record), Hunan (new Chinese provincial record).

Specimens examined: CHINA: Fujian: $10^{*}$ (FLWC), Sanming, VII.2014, Bi-Huang Zhan leg.; $10^{\star}$ (FLWC), Mangdang Mount, Nanping, 5.VII.2009, no leg.; $10^{\star}$, 2 우 우 (FLWC), Damo Mount, Sanming, 15.VI.2018, Xin Liu leg. Guangdong: 3 우 (FLWC), Houzidong Mount, Wengyuan County, Shaoguan, 18-19.V.2018, Jian-Wei Chen leg. Guangxi: $2 o^{\star} o^{\star}, 6$ ㅇ (FLWC), Dayao Mount, Jinxiu County, Laibin, Guangxi, China, VII.2015, Bao-Sheng Su leg.; 1 ㅇ (FLWC), Ziyuan County, Guilin, Guangxi, China, VII-VIII.2019, Wen Jin leg. Guizhou: 1 ㅇ (FLWC), Shunchangxiang, Shuicheng County, Shuicheng, Liupanshui, China, alt. 1100 m, 22.V.2019, Gui-Qiang Huang leg. Hunan: $1 \sigma^{\star}, 1$ 우 (FLWC), Mount Yangming, Yongzhou, Hunan, China, alt. 900 m, 1.VII.2019, Yu-Tang Wang \& Yi-Ting Zhong leg. Zhejiang: $1 \sigma^{\star}, 1$ 우 (FLWC), Kuocang Mount, Taizhou, Zhejiang, China, VII.2015, Zhao-Yang Tang leg.

Remarks: This species is currently only known from China. Previously it was recorded from Fujian, Guangdong and Guangxi (MachatschKe 1955; Lin 1993; Lin 2002b; ZoRn 2011; ZoRN \& BEZDĚK 2016). Here we newly report this species from Guizhou and Hunan.

\section{Anomala siamensis (NonfRIED, 1891)}

(Figs 20, 46-49)

Distribution: Thailand; China (new country record): Yunnan; Laos (new country record).
Type material: Syntypes[?]. 1 (MFNB), Coll. Nonfried. Siam / Aprosterna siamensis Type Nonfr. [Ohaus's handwriting] / Anomala siamensis Bang-Pain Type A. F. Nonfried [possibly Nonfried's handwriting]; $10^{\star}$ (MFNB), Siam Nonfried / Heteroplia n. sp. / Aprosterna siamensis Cotype Nonfr. [Ohaus's handwriting].

Note: It is not entirely certain that these two specimens from Ohaus's collection are real syntypes of Aprosterna siamensis Nonfried, 1891 because they do not bear Nonfried's type label (compare with the label shown in Horn et al. 1990).

Other specimens examined: CHINA: Yunnan: $10^{\text {t }}$ (FLWC), Menglun, Xishuangbanna, 15.IV.2019, Zi-Chun Xiong leg.; LAOS: 1 \% (CZGG), Laos NW, Nam Tha NPA, $65 \mathrm{~km}$ nw Luang Nam Tha, $1050 \mathrm{~m}$, 8-15.IV.2010, S. Murzin leg.; 1 क (PPPC), LAOS centr 27.IV.-1.V.1997. $70 \mathrm{~km}$ NE Vientiane, Ban Phabat env., $150 \mathrm{~m} \mathrm{~N} 18^{\circ} 16.1^{\prime} \mathrm{E} 103^{\circ} 10.9^{\prime} \mathrm{E}$. Jendek \& O. Sausa leg.; $10^{\star}$ (PPPC), S Laos Attapu, Nong Lom (lake), 18-30.IV.1999, $800 \mathrm{~m}, 15^{\circ} 02^{\prime} \mathrm{N}, 106^{\circ} 35^{\prime} \mathrm{E}, \mathrm{M}$. Strba leg. THAILAND: $1 \sigma^{\top}$ (PLPC), THAILAND: Phayao Prov. Naresuan University; lights on campus; 19. April 2003; L-467 Vitheepradit, Prommi, Setaphan; $10^{*}$ (AHPC), Thailand, $9.11 .199212 \mathrm{~km}$ SW Pak-Chong $130 \mathrm{~km}$ NO Bangkok Mischobstplantage, $400 \mathrm{~m}$ Lichtfang, Thielen leg.; 1 o (PLPC), Thailand, Ubon Ratchathani Prov., Phu Chong $\mathrm{Na}$ Yoi National Park, Kaeng Ka Loa, 11. April 2004, $182 \mathrm{~m}, 14^{\circ} 26.178^{\prime} \mathrm{N} 105^{\circ} 16.631 \mathrm{E}$, Sites \& Vitheepradit leg.; 3 o $^{\star}, 1$ ㅇ (CZGG), NW Thailand, Mae Hong Son, Pan Huai Po, 1600 m, 9-16.V.1991, leg. P. Pachlátko; $10^{\star}$ (CZGG), NW Thailand, Chom Thong, 24-27.IV.1991, leg. Pacholátko.

Remarks: PAUlian (1959) misidentified Anomala anguliceps as A. siamensis, but later ZoRn et al. (2017) corrected this identification based on the male genitalia illustrated in Paulian (1959). Also, YANG (1989) misidentified A. anguliceps as $A$. siamensis when reporting this species from Guizhou, China. Both species mentioned above were also confused by $\operatorname{Lin}(1987,1992,2002 b)$, who consequently described A. corneola LIN, 2002, which was subsequently synonymized with $A$. anguliceps by Prokofiev (2014).

In fact, Anomala siamensis is similar to A. bilobata Arrow, 1912 and A. fissilabris Arrow, 1912 rather than $A$. anguliceps. Both $A$. bilobata and $A$. fissilabris have a bilobed clypeus in males, which is the same in males of A. siamensis, while the clypeal front margin is straight in A. anguliceps. Anomala siamensis was previously known only from Thailand, here this species is newly recorded from China and Laos. 


\section{Anomala xanthoptera BLANCHARD, 1851}

(Figs 21, 50-52)

Distribution: India; Nepal; Pakistan; China (new country record): Yunnan.

Type material: Syntype of Anomala prasinicollis BATES, 1891 [junior subjective synonym of Anomala xanthoptera]. $10^{\star}$ (MFNB), PUNJAB Kulu Distr. / Euchlora prasinicollis Cotype Bts. [Ohaus's handwriting] / Anomala prasinicollis Bates [Bates's handwriting].

Other specimens examined: CHINA: Yunnan: $1 \sigma^{*}$, 2 우 (FLWC), Nabang, Yingjiang County, Dehong, 17.II.2016, Hao Xu \& Jian-Yue Qiu leg. INDIA: $34 \sigma^{\star} \sigma^{\star}$, 8 우 으 (CZGG, ZMH), Indien, Bhimtal, F. Smetacek leg., Museum Hamburg, Eing. Nr. 3/1977; 1 ○ (SMTD), Sikkim India. NEPAL: $10^{*}$ (CZGG), Nepal, Narayani Prov. Sauraha Ufer Rapti River 180 m, 273' $80^{\prime \prime N}$, 84²9'49"E, 18.IV.2000, leg. A. Skale, LF [collected at light]; 1 o $^{\star}$ (CZGG), Nepal-Himalaya, Myagdi distr., Babichor-Beni, 16.III.1994, 900-1000 m, leg. D. Ahrens. PAKISTAN: $10^{\top}$ (HNHM), Pakistan, Kashmir, Himalaya, Mts. $20 \mathrm{~km} \mathrm{~S} \mathrm{Muzaffarabad,} \mathrm{Nara} \mathrm{village,} 750 \mathrm{~m}, 73^{\circ} 29^{\prime} \mathrm{E}$, 3401'N, 20.V.1998, leg. M. László \& G. Ronkay.

Remarks: This species was previously known from India, Nepal and Pakistan (BAtes 1891; Arrow 1917; Zorn \& BEZDĚK 2016), and is here newly recorded in China based on three specimens collected from Yunnan.

\section{Anomala zornella ProKOFIEV, 2015}

Distribution: Laos; Vietnam; China (new record): Yunnan.

Specimens examined: CHINA: Yunnan: $120^{\star} \sigma^{\star}, 4$ 우 우 (FLWC), Jinping County, Honghe, V-VI.2018, YunChuan Xu leg.; 3 o $^{\star} \sigma^{\star}$ (FLWC), Lvchun County, Honghe, 20.IV.2019, Zi-Chun Xiong leg.

Remarks: Anomala zornella was originally described from Laos, Myanmar and Vietnam (Prokofiev 2015). Here, we newly record this species from Yunnan, China. Specimens from both Jinping County and Lvchun County have reddish legs, which are also present in the specimens collected from Laos and Sa Pa, Vietnam, whereas specimens from Tam Dao, Vietnam, have greenish leg. (Prokofiev 2015; Zorn et al. 2017).

\section{Discussion}

The species described in this work, i.e. Anomala xiongi sp. nov., is easily recognized by the conspicuous elytral setae. Except for the pygidium and propygidium, a sometimes present row of setae along the elytral margin, and isolated tactile setae on head and pronotum, distinct setation of the dorsal surface is a rather uncommon character among most genera of Anomalini (MACHATSCHKE 1957). However, dorsal setation is found in the majority of species of the subtribe Anisopliina, in Isopliina, in several genera of the subtribe Popilliina, and, within the subtribe Anomalina, in many species of the genera Anoplanomala Arrow, Asiopertha Medvedev, Blitopertha ReITter, Cyriopertha ReITter, Epectinaspis Blanchard, Exomala Reitter, Hontoriella Escalera, Phyllopertha StePhens, Proagopertha ReItTer, Pseudotrigonocnemis KeITH, and Trigonocnemis KrAATz. In the genus Anomala, setose elytra are a rarely observed character, which has incurred special attention in some taxonomic research (FILIPPINI et al. 2013; LiN 1996; MiYake 1994). Dorsally setose elytra are present in Asian and New World Anomala species but not in Afrotropical species and seem to appear in clusters of otherwise unrelated species-group, e. g. A. hirsutulagroup (Lin 1996; Machatschke 1957; Miyake 1994), A. trapezifera-group (FILIPPINI et al. 2015). Notably, the trapezifera-group comprises species with and without dorsal setation (FILIPPINI et al. 2015), which demonstrates the plasticity of this character. The presence of microscopic hairs found in the elytral and pronotal punctures of many Anomala species suggests that a distinctly setose dorsal face evolves by enlargement of an already existing structure, which makes it more likely to appear independently in unrelated groups.

In Asian Anomala species, setose elytra are only found in the 21 species of the hirsutula-group, which occur in southern China, Laos, Myanmar, Thailand, and Vietnam (Lin 1996, Machatschke 1957, MiYake 1994, 1996; KeIth 2008), and also in A. dayaoshanensis WANG, 2020 (China: Guangxi), A. graminea OHAus, 1905 (southern China, Vietnam), A. herbacea ZorN, 2017 (Vietnam), A. inopinata OHAus, 1914 (Philippines), A. obesa CANDÈze, 1869 (Philippines), A. ordinata ZorN, 2017 (Vietnam), A. sordidula Sharp, 1881 (West Malaysia), A. xiongi sp.n., and A. yunnana (FAIRmaire, 1886) (southern China, Vietnam). Except for the species within the hirsutula-group and for A. graminea and A. yunnana, which both share a very similar apomorph structure of the aedeagus, none of the other species seem to be related to each other. The elytral setae in Asian Anomala are usually grayish to yellowish, short and adpressed to semierect. They are confined to the irregular punctures and never seem to be present in strial punctures. Anomala xiongi sp. n. is the only known Anomala species with long erect setae confined to the primary costae of elytra. However, external morphology and shape of aedeagus suggest that the closest relatives of $A$. xiongi sp. nov. are species without dorsal setosity. Thus, this character is suitable for species diagnosis, but seems to have limited significance for higher level systematics in Anomalini. 


\section{Acknowledgements}

We wish to express our sincere gratitude to $\mathrm{Zi}$-Chun Xiong (Yunxian, Yunnan) for collecting the majority of the type material of $A$. xiongi WANG \& ZorN new species, as well as to Shao-Fu Chen (Yingjiang, Yunnan), Andreas Hetzel (Münster), Paul K. Lago (Oxford, Mississippi), Petr Pacholátko (Brno), Ai-Min Shi, YunChun Li, and Yuan-Yuan Ma (CWNU), Matthias Seidel (Hamburg), Chang-Chin Chen (Tianjin), and Ming-Zhi Zhao (Guangzhou, Guangdong) for providing material from their collections for this study. Moreover, we are very grateful to the collection managers and curators Ottó Merkl ${ }^{\dagger}(\mathrm{HNHM})$, Antoine Mantilleri, Olivier Montreuil and Thierry Deuve (MNHN), Matthias Hartmann (NMEG), Martin Fikáček, Jiří Hájek and Lukáš Sekerka (NMPC), Olaf Jäger (SNSD), Johannes Frisch, Bernd Jäger and Joachim Willers (MFNB), and Martin Husemann and Matthias Seidel (ZMH) for providing access to the collections of their institutions. We also sincerely thank Hao Xu and Jian-Yue Qiu (MYTC), Lu Qiu (Southwest University, Chongqing), Zhi-Wei Dong (Kunming, Yunnan), Gui-Qiang Huang (Liupanshui Normal University, Guizhou), Zhi-Hao Qi (Wuyishan, Fujian), Zhao-Yang Tang (Shenzhen, Guangdong), Huang-Shun Xi (Shanghai, Shanghai), Yun-Chuan Xu (Yuxi, Yunnan), Zheng Zhou (Chuxiong, Yunnan), and Chao Zhou (Chengdu, Sichuan) for collecting Anomala specimens for this study.

\section{References}

Arrow, G. J. 1912a: Some new species of the lamellicorn genus Anomala from Sikkim, North India. - The Annals and Magazine of Natural History, including Zoology, Botany, and Geology, series 8, 9: 72-84.

Arrow, G. J. 1912b: Descriptions of some new Burmese Species of Ruteline Coleoptera belonging to the genus Anomala. - The Annals and Magazine of Natural History, including Zoology, Botany, and Geology, series 8, 10: 327-340.

Arrow, G. J. 1917: The fauna of British India, including Ceylon and Burma. Coleoptera Lamellicornia part II (Rutelinae, Desmonycinae, and Euchirinae). - Taylor \& Francis, London: $387 \mathrm{pp}+\mathrm{v}$ pl.

BATES, H. W. 1891: Coleoptera from Kulu in N.W. India. - The Entomologist 24 (Supplement): 7-23.

CANDÈzE, E. C. A. 1869: Diagnoses de quelques Rutélides nouvelles. - ColeopterologischeHefte 5:41-45.- https:// archive.org/stream/coleopterologisc115haro\#page/41/ mode/1 up [accessed 2020/11/15].

Fairmaire, L. 1886: Descriptions de Coléoptères de l'intérieur de la China. - Annales de la Société Entomologique de France, 6. Ser. 6: 303-356. - https://www. biodiversitylibrary.org/page/34026721\#page/309/ mode/1up [accessed 2020/11/15].
Filippini, V.; Mico, E. \& Galante, E. 2013: Redescription of Anomala eucoma BATEs, 1888 and a description of three new species from Costa Rica. - Zootaxa 3670 (2): 255-273.

Filippini, V.; Mico, E. \& Galante, E. 2015: Anomala trapezifera species-group: a burst of diversity (Coleoptera: Scarabaeidae: Rutelinae). - Annales de la Société entomologique de France (N.S.): 51 (2): 93-139.

Frey, G. 1975: Ergebnisse der Bhutan-Expedition 1972 des Naturhistorischen Museums in Basel. Coleoptera: Fam. Scarabaeidae Subf. Rutelinae. - Entomologica Basiliensia 1: 243-247.

Horn, W.; Kahle, I.; Friese, G. \& Gaedike, R. 1990: Collectiones entomologicae. Ein Kompendium über den Verbleib entomologischer Sammlungen der Welt bis 1960. - Akademie der Landwirtschaftswissenschaften der Deutschen Demokratischen Republik, Berlin: $473 \mathrm{pp}$.

Huang, G. Q. \& Wang, F. L. 2019: Two new and one newly recorded species of Anomala SAMOUELLE, 1819 (Coleoptera: Scarabaeidae: Rutelinae) from Yunnan, China. - Zootaxa 4706 (2): 366-374.

Keith, D. 2008: Description d'une nouvelle espece d'Anomala du Myanmar. - Symbioses 22: 71-72.

KRAJČíK, M. 2012: Checklist of the World Scarabaeoidea. - Animma. X Supplement 5: 1-278.

LiN, P. 1981: Coleoptera: Rutelidae.: 355-387. - In: TAN, J.-J. \& WANG, S.-J. (eds.): The Series of the Comprehensive Scientific Expedition to the QinghaiXizang Plateau. Insects of Xizang. Volume 1. - Science Press, Beijing: i-xi + 600 pp. (in Chinese and English).

LIN, P. 1987: Coleoptera: Rutelidae.: 566-570. - In: HuAng, F. S. (ed.): Forest Insects of Yunnan. - Yunnan Science \& Technology Press, Kunming: i-ix + 1622 pp. (in Chinese).

LIN, P. 1988: Coleoptera: Rutelidae.: 249-265. - In: HuANG, F. S.; WANG, P. Y.; YIN, W. Y.; YU, P. Y.; LEE, T. S.; YANG, C. K. \& WANG, X. J. (eds.): Insects of Mt. Namjagbarwa region of Xizang. - Science Press, Beijing: i-xii + 621 pp. (in Chinese and English).

Lin, P. 1992: Coleoptera: Rutelidae.: 503-524. - In: Chen, S. (ed.): Insects of the Hengduan Mountains region. Volume 1. - Science Press, Beijing: i-xii + 865 pp. (in Chinese and English).

Lin, P. 1993: A systematic revision of the China Mimela: (Coleoptera: Rutelidae). - The Publishing Company of Zhong Shan University, Guangzhou: 106 pp + xxii pl. (in Chinese and English).

LIN, P. 1996: New species of Anomala hirsutula species group from China and discussion on their taxonomic problems. - Entomotaxonomia 18: 157-169.

Lin, P. 1999: New species of the genus Anomala SAmouelle (Coleoptera: Rutelidae) collected in Yunnan, China. - Entomotaxonomia 21: 157-176. (in Chinese and English). 
Lin, P. 2002a: Coleoptera Rutelidae: 345-355. - In: HuAng, F. (ed.): Forest insects of Hainan. - National Natural Science Foundation of China, Beijing: xv + 1064 pp +31 pls. (in Chinese and English).

Lin, P. 2002b: Rutelidae.: 387-427. - In: HuAng, B. (ed.): Fauna of Insects of Fujian Province of China. Volume 6. - Fujian Science \& Technology Press, Fuzhou: 894 pp. (in Chinese and English).

Machatschкe, J. W. 1957: Coleoptera Lamellicornia Fam. Scarabaeidae Subfam. Rutelinae 2. Genera Insectorum Fasc. 199 (B). - Imprimerie et Editions Mercurius, Antwerp, Belgium: 219 pp + vi pls.

Miyake, Y. 1994: New or little known Scarabaeid Beetles from Southeast-Asia II. - Special Bulletin Essa entomological Society 2: 139-156.

MiYAKe, Y. 1996: New or little known Scarabaeid Beetles from Southeast Asia III. - Lamellicornia 11: 33-54.

Nonfried, A. F. 1891: Weitere Beiträge zur Käferfauna von Süd-Asien und Neu-Guinea. - Berliner entomologische Zeitschrift 36: 359-380. - https://www. biodiversitylibrary.org/item/101306\#page/411/ mode/1up [accessed 2019/10/3].

OHAus, F. 1905: Beiträge zur Kenntnis der Ruteliden. Deutsche Entomologische Zeitschrift 1905: 81-99. - https://archive.org/stream/deutscheentomol00natugoog\#page/n87/mode/1up [accessed 2020/11/15].

Ohaus, F. 1914a: XV. Beitrag zur Kenntnis der Ruteliden. - Stettiner Entomologische Zeitung 75: 193-217. - https://www.biodiversitylibrary.org/item/ 35652\#page/205/mode/1up [accessed 2019/10/3].

Ohaus, F. 1914b: Zweiter Nachtrag zur Kenntnis der Ruteliden der Philippin. Inseln. - Stettiner entomologische Zeitung, Stettin 75: 185-193. - https:// archive.org/stream/stettinerentomol751914ento\#page/n196/mode/1 up [accessed 2020/11/15].

Ohaus, F. 1915: XVII. Beitrag zur Kenntnis der Ruteliden. - Stettiner entomologische Zeitung 76: 88-143. - https://www.biodiversitylibrary.org/page/ 8826074\#page/314/mode/1up [accessed 2017/10/13].

OHAus, F. 1937: Neue Ruteliden des Naturhistorischen Riksmuseum in Stockholm. - Arkiv för Zoologi 30B: $1-7$.

Paulian, R. 1959: Coléoptères Scarabéides de l'Indochine (Rutélines et Cétonines). - Annales de la Société entomologique de France 128: 35-136. http://gallica.bnf.fr/ark:/12148/bpt6k6142156w/f14 [accessed 2017/06/26].

Prokofiev, A. M. 2014: New and noteworthy scarab beetles from Asia and America (Coleoptera Lamellicornia). - Calodema 330: 1-25. - https://www.zin. ru/ Animalia/Coleoptera/pdf/prokofiev_2014_new_and_ noteworthy_scarab_beetles.pdf [accessed 2017/10/12].

Prokofiev, A. M. 2015: New Anomala Samouelle, 1819 from South-East Asia (Coleoptera: Scarabaeidae: Rutelinae). - Russian Entomological Journal 24: 37-59. - http://kmkjournals.com/upload/PDF/ REJ/24/ent 24_1_037_059_Prokofiev_for_Inet.pdf [accessed 2017/06/26].
Sabatinelli, G. 1997: Nuovo Adoretosoma, Anomala, Callistethus, Ischnopopillia e Mimela del Himalaya e del Sud-Est Asiatico. - Lambillionea 97 (2): 242-258.

SHARP, D. 1881: Descriptions of new species of Melolonthini and Rutelini, collected in the Island of Sumatra during the scientific Sumatra-Expedition Notes from the Leyden Museum 3: 219-242. - https:// archive.org/stream/notesfromleydenm03rijk\#page/ n240/mode/1up [accessed 2020/11/15].

YANG, C. J. 1989: Scarabaeoidea. - In: Guo, Z. Z. (ed.): The Agricultural and Forestry Insect Fauna of Guizhou, Volume 2. - China: Guizhou People's Publishing House, Guiyang: 88 pp. (in Chinese).

WANG, F. 2020: Nine new species of the genus Anomala from China - Kogane 23: 61-75.

ZhaO, M. Z. 2019: A new species of the genus Anomala from southern China (Coleoptera: Scarabaeidae: Rutelinae). - Acta Entomologica musei Nationalis Pragae 59 (2): 481-489. - DOI: 10.2478/aemnp-20190037.

ZorN, C. 2005: Taxonomical acts in the Anomalini initiated during the preparation of the "Catalogue of Palaearctic Coleoptera". - Acta Societatis Zoologicae Bohemicae 68: 310-328.

Zorn, C. 2007: Taxonomic revision of the Anomala cuprascens - species group of Sulawesi and the Papuan region: The species with unidentate protibiae (A. chlorotica-subgroup) (Coleoptera: Scarabaeidae: Rutelinae). - Arthropod Systematics \& Phylogeny 65: 25-71. - http://www.senckenberg.de/files/content/ forschung/publikationen/arthropodsystematics/ asp_65_1/asp_65_1_zorn_25-71.pdf [accessed 2017 /06/26].

Zorn, C. 2011: New species of the genus Anomala SAMOUElle from mainland South East Asia and South China. - Stuttgarter Beiträge zur Naturkunde A, Neue Serie, 4: 297-312. - http:// www.naturkundemuseum-bw.de/sites/default/files/ publikationen/serie-a/ns04-15zorn.pdf [accessed 2017/06/26].

Zorn, C. \& BEZDĚK, A. 2016: Rutelinae.: 317-358. - In: LöBL, I. \& LöBL, D. (eds.): Catalogue of Palaearctic Coleoptera. Volume 3. Scarabaeoidea - Scirtoidea Dascilloidea - Buprestoidea -Byrrhoidea. Revised and updated edition. - Brill, Leiden, Boston: 317-358.

Zorn, C.; Kobayashi, H. \& WAdA, K. 2017: Notes on the genus Anomala Samouelle, 1819 (Coleoptera, Scarabaeidae, Rutelinae) in Vietnam and neighboring regions: eight new species and faunistic records. - Contributions to Entomology $67(2)$ : 325-352. - DOI: https://doi.org/10.21248/contrib. entomol.67.2.325-352. 

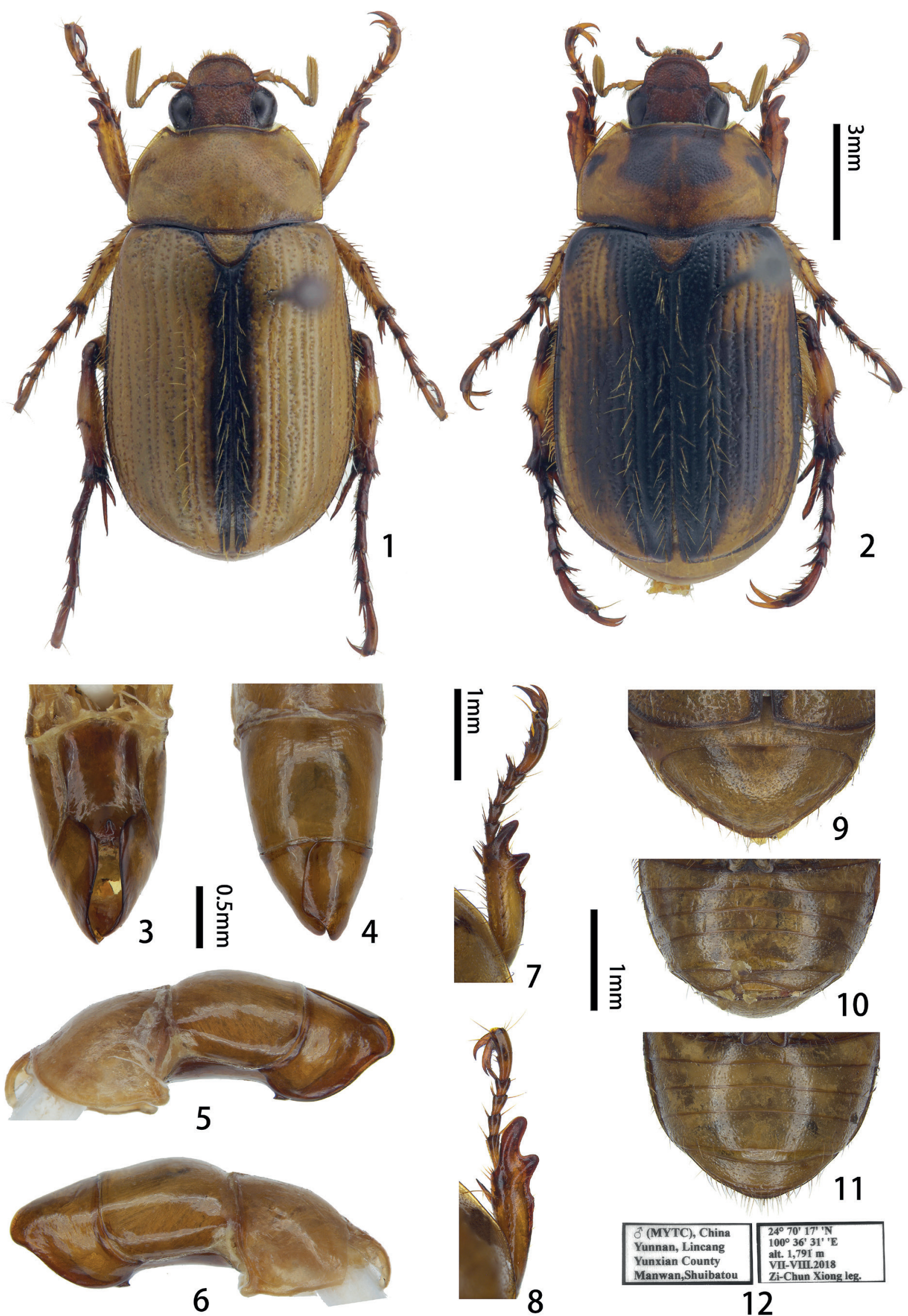

Figs 1-12: Anomala xiongi WANG \& Zorn, new species - 1, 3-7, 9, 10. holotype, male. 2, 8, 11. paratype, female. 1, 2. habitus. 3-6. male genitalia. 7, 8. protibial and protarsal. 9. pygidium. 10, 11. abdominal ventrites. 12. provenance label of holotype. 1, 2, 4. dorsal view. 3. ventral view. 5. lateral view from left. 6. lateral view from right. 

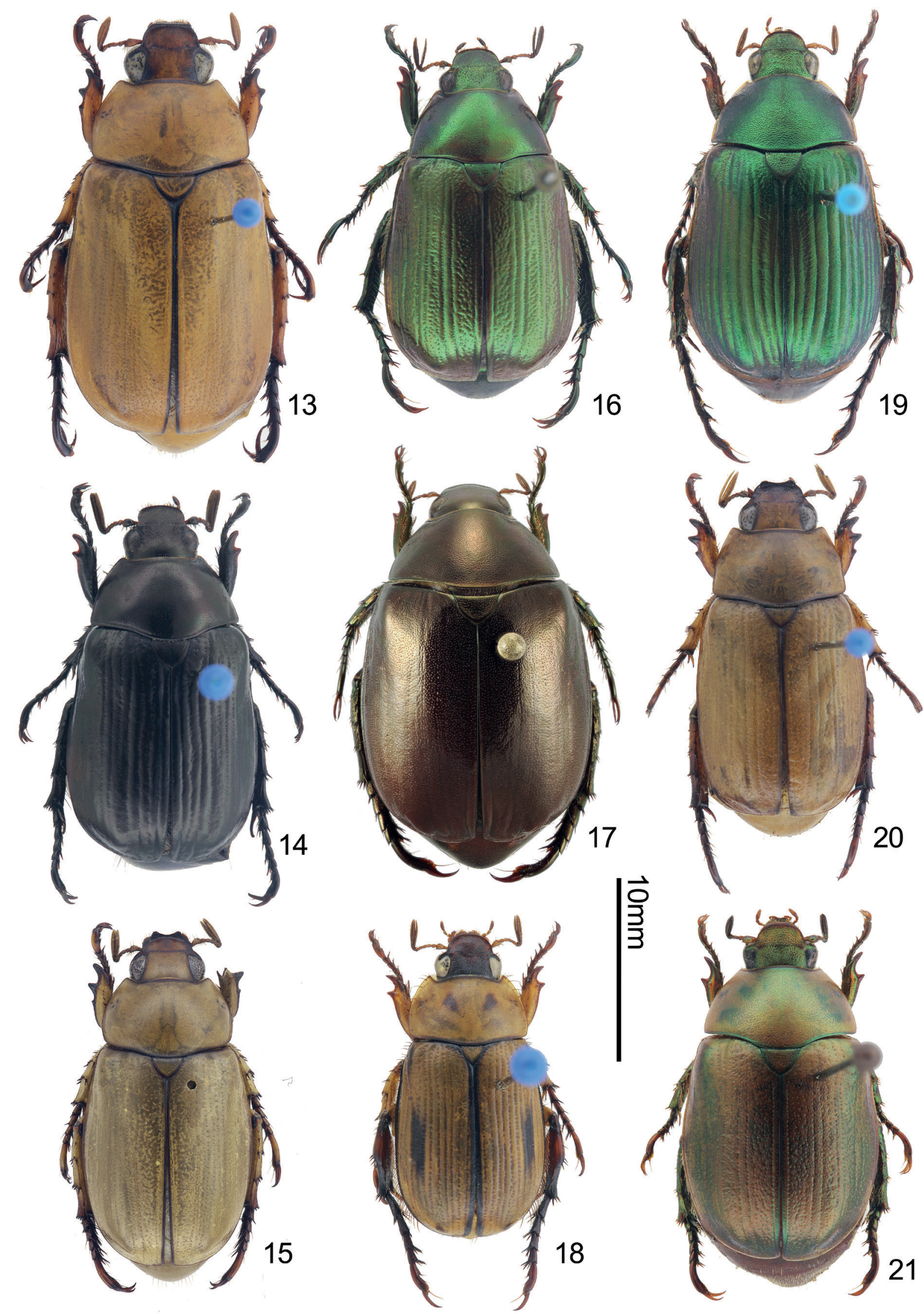

Figs 13-21: Male habitus of Anomala spp., male. - 13. A. anguliceps (Emei Mount, Sichuan). 14. A. anthracina (Gongshan County, Yunnan). 15. A. bilobata (Yingiiang County, Yunnan). 16. A. callifera (Gongshan County, Yunnan). 17. A. cantorioides (Mengla, Yunnan). 18. A. fusitibia (Yingjiang County, Yunnan). 19. A. kuatuna (Jinxiu County, Guangxi). 20. A. siamensis (Menglun County, Yunnan). 21. A. xanthoptera (Yingjiang County, Yunnan). 

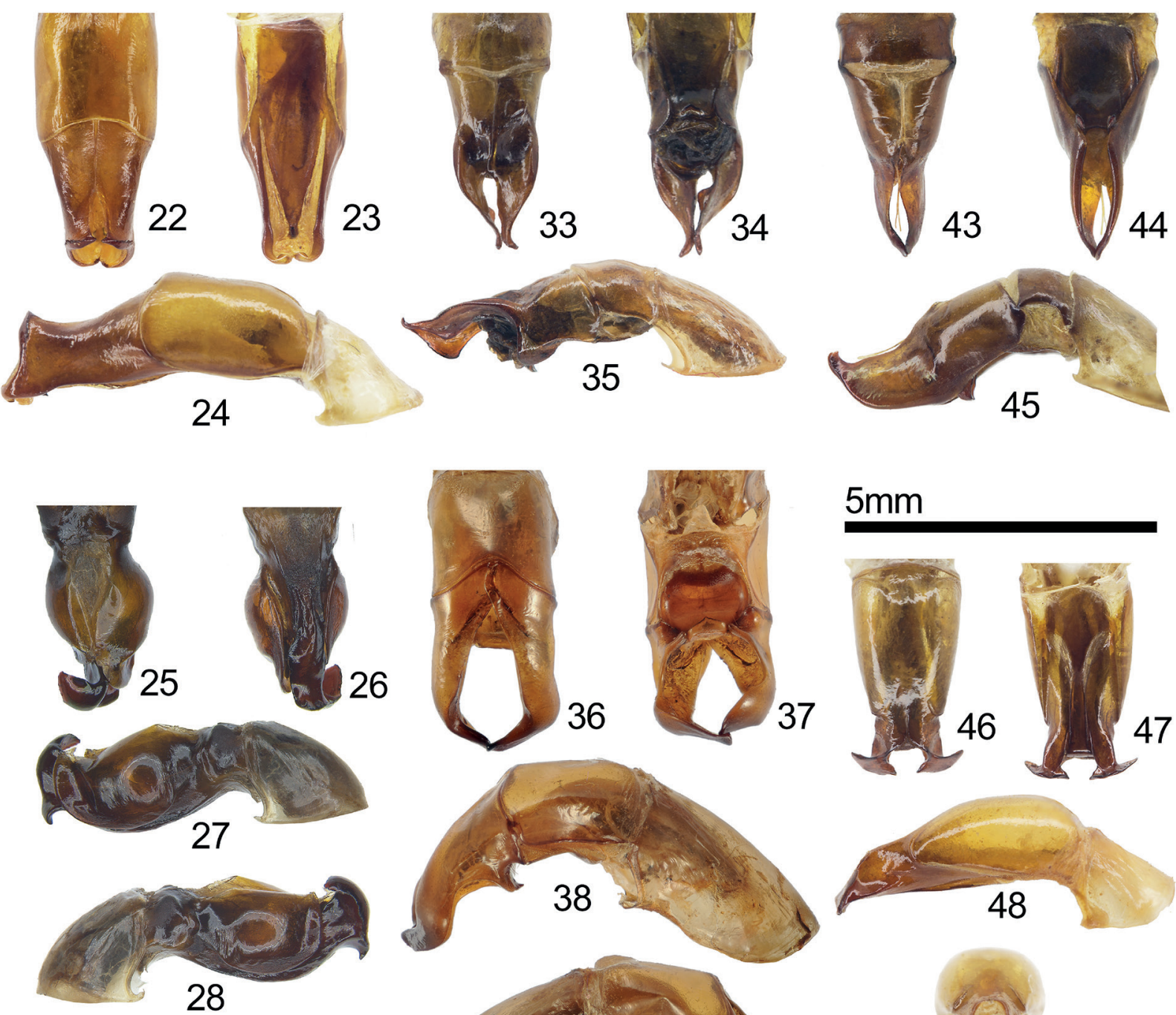

\section{$5 \mathrm{~mm}$}
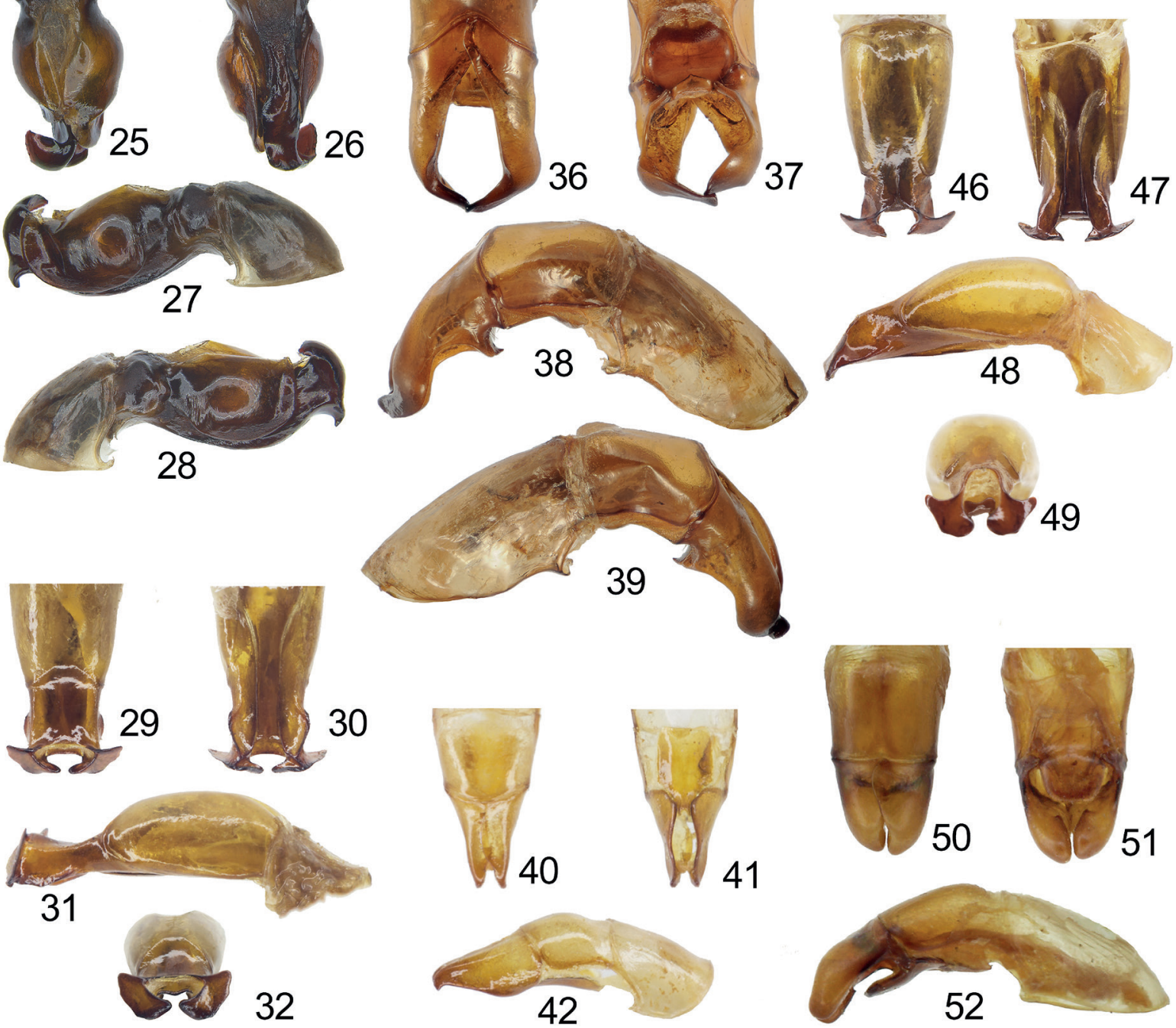

32
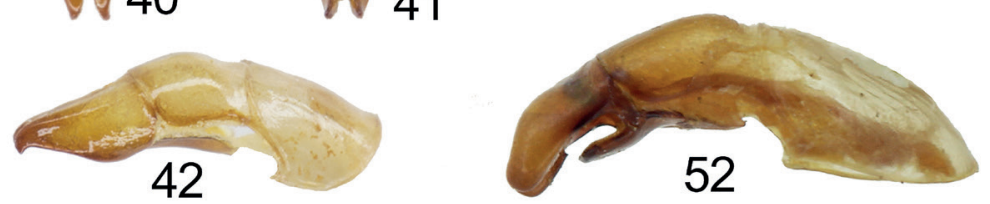

Figs 22-52: Aedeagus of Anomala spp., male. - 22-24. A. anguliceps (Emei Mount, Sichuan). 25-28. A. anthracina (Gongshan County, Yunnan). 29-32. A. bilobata (Yingjiang County, Yunnan). 33-35. A. callifera (Gongshan County, Yunnan). 36-39. A. cantorioides (Mengla, Yunnan). 40-42. A. fusitibia (Yingjiang County, Yunnan). 43-45. A. kuatuna (Jinxiu County, Guangxi). 46-49. A. siamensis (Menglun County, Yunnan). 50-52. A. xanthoptera (Yingjiang County, Yunnan). 22, 25, 29, 33, 36, $40,43,46,50$. dorsal view. $23,26,30,34,37,41,44,47,51$. ventral view. $24,28,31,35,38,42,45,48,52$. lateral view from right. 28, 39. lateral view from left. 32,49 . frontal view. 
Fig. 53: Distribution map of Anomala xiongi WANG \& ZoRN, new species.

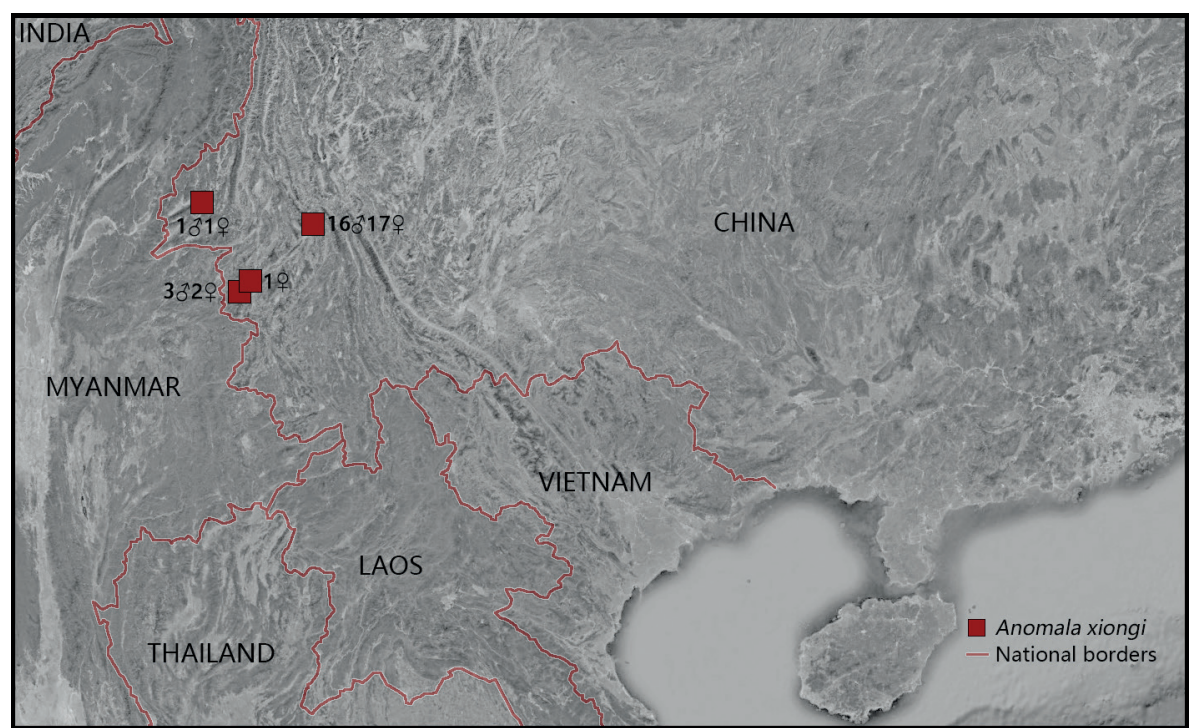

